\title{
Vitamin Uptake Alteration
}

National Cancer Institute

\section{Source}

National Cancer Institute. Vitamin Uptake Alteration. NCI Thesaurus. Code C40815.

A finding indicating a deficiency or excess in absorption or assimilation of one or more vitamins. 